\title{
Impact of supervised exercise training on pulmonary function parameters, exercise capacity and Irisin Biomarker in Interstitial lung disease patients
}

\author{
Rahma Mohammad Alyami ${ }^{1}$, Abdulrahman Mohammed Alhowikan², \\ Abdullah Rashed Alharbi3 ${ }^{3}$ Ghada AL-Nafisah ${ }^{4}$
}

\begin{abstract}
Objectives: To assess the impact of supervised exercise training (SET) on pulmonary function Parameters, exercise capacity and Irisin biomarker in Interstitial Lung Disease (ILD) patients.

Methods: Ten (10) patients with ILD and 18 healthy controls of age between 30-40+ years were selected for 8-week SET program. Before and after SET all subjects performed exercise capacity six minutes' walk test (6MWT), heart rate (HR) changes were recorded, shortness of Breath Respiratory Questionnaire (SOBQ) was obtained and Irisin levels were measured by Enzyme-Linked Immunosorbent Assay (ELISA). This interventional study was carried out at Department of Physiology, Faculty of Medicine, King Khalid University Hospital, King Saud University, Riyadh, Saudi Arabia, from October 2018 to February 2019.

Results: Mean six minutes' walk distance (6MWD) was $395 \pm 68.4 \mathrm{~m}$ at 1 st visit increased significantly $(\mathrm{p}=0.001)$ to $458.8 \pm 87.1$ mat 15 visit. However, 6 MWD values found significantly higher in controls $(517.4 \pm 84.1 \mathrm{~m}$; $561.7 \pm 81.6 \mathrm{~m} ; \mathrm{p}=0.013$ ) than ILD patients. Overall change (difference between post and pre exercise) in $\mathrm{HR}$ value was recorded lower in ILD patients (30-35 bpm) as compared to controls (40-45 bpm). Moreover, ILD patients had overall higher SOBQ score than controls. Pre SET Irisin levels of ILD patients $(4.24 \pm 1.73$ $\mathrm{pg} / \mathrm{ml})$ and controls $(3.43 \pm 1.04 \mathrm{pg} / \mathrm{ml})$ were found unchanged after SET $(4.48 \pm 2.02 \mathrm{pg} / \mathrm{ml}, 3.39 \pm 1.41 \mathrm{pg} / \mathrm{ml}$, $p=0.677, p=0.093$ ) respectively. However, patients Irisin values were found higher as compared to controls before and after SET.

Conclusion: Exercise capacity and Dyspneain patients with ILD were improved after 8-week of SET program. No major changes in Irisin levels among patients with ILD and controls were observed. Additional research requires to be carried out on large number of subjects to deterMinutese the advantages of exercise in ILD.
\end{abstract}

KEYWORDS: Supervised exercise training, Irisin, Interstitial Lung Disease.

doi: https://doi.org/10.12669/pjms.36.5.1795

How to cite this:

Alyami RM, Alhowikan AM, Alharbi AR, AL-Nafisah G. Impact of supervised exercise training on pulmonary function parameters, exercise capacity and Irisin Biomarker in Interstitial lung disease patients. Pak J Med Sci. 2020;36(5):1089-1095. doi: https://doi.org/10.12669/pjms.36.5.1795

This is an Open Access article distributed under the terms of the Creative Commons Attribution License (http://creativecommons.org/licenses/by/3.0), which permits unrestricted use, distribution, and reproduction in any medium, provided the original work is properly cited.

Correspondence:

Abdulrahman Mohammed Alhowikan, PhD.

Department of Physiology,

Faculty of Medicine,

King Saud University,

P. O Box 2925, Riyadh 11461 ,

Saudi Arabia.

Email: ahowikan@ksu.edu.sa

* Received for Publication:

* Revision Received:

* Accepted for Publication:
October 9, 2019

May 14, 2020

May 20, 2020

\section{INTRODUCTION}

In both healthy subjects and patients with chronic diseases, physical fitness and regular exercise have been shown to improve functional health and reduce mortality risk. ${ }^{1}$ Exercise training (ET) and rehabilitation programs in patients with chronic respiratory disease have been reported to improve signs and exercise ability, but the degree and period of the outcome in precise situations need to be completely explained. ${ }^{2}$ Exercise 
training is an essential factor of evidence-based controlling programs for several long-lasting cardiac and respiratory disorders. There is a substantial indication that exercise activity encourages major improvement in exercise tolerance and useful rehabilitation for patients with interstitial lung disease (ILD). ${ }^{3}$

The Interstitial Lung Disease isa chronic lung condition categorized by dyspnea on exertion and deprived health related quality of life. ${ }^{4}$ The role of exercise training and pulmonary rehabilitation programmes in the ILD patients is still uncertain.A number of exercise training studies recommend that pulmonary rehabilitation programs that contain exercise training may recover functional capacity and quality of life in the ILD, ${ }^{5}$ however, there is noticeable difference in results between individuals. ${ }^{6}$ Physical exercise offers potential as a useful treatment for subjects with ILD, with advancements in six-minute's walk distance (6MWD), dyspnoea, and exercisecapacity. ${ }^{7}$ Recently Bonini et al. ${ }^{8}$ reviewed and explained the respiratory physiology of ILD, together at rest and throughout exercise. However according to clinical practice guidelines the quality of the data supporting the use of exercise training ${ }^{9}$ islimited and that the longstanding benefits are not clear. ${ }^{10}$

In sports biomarkers are main factors to evaluate the effect of exercise on altered systems, tissues and organs. Moreover, blood biomarkers can be used as tool to assess the effect of exercise training on patients with ILD. In response to physical exercise, muscle tissue secrete myokine called Irisin, and it is believed that these myokines are involved in physical activity induced positive healthresults. ${ }^{11}$ However in humans, the regulation of Irisin by exercise is not clear. Huh et al. ${ }^{12}$ examined the effect of acute and chronic whole-body vibration exercise on circulating irisin levels in young healthy subjects. In previous studies Irisin has been recommended to mediate the useful effects of exercise. Thus, Irisin could be a balanced biomarker for diseases linked with physical inactivity..$^{13}$ However, many studies have reported increase in Irisin blood concentration after acute exercise training. ${ }^{14} \mathrm{~A}$ number of reports have established the improved circulating Irisin in response to acute bouts of exercise, mostly using treadmill or bicycleexercise. ${ }^{15}$ It remains to be elucidated whether other types of exercise can increase irisin acutely or after a period of training. Moreover, whether main physiological ordemographic variables play a part in estimation of irisin's response to exercise remains to be clarified.
The main object of this study was to assess the impact of supervised exercise training (SET) on pulmonary function Parameters, exercise capacity and Irisin biomarker in ILD patients.

\section{METHODS}

Interstitial Lung Disease (ILD) patients ( $\mathrm{n}=10$, male $=3$; female 7) with stable condition and healthy controls $(n=18$, male $=7$; female $=11)$ aged between 30-40+ years were recruited in this interventional design (single-arm clinical trial (SACT) study, conducted at Department of Physiology, Faculty of Medicine, King Khalid University Hospital (KKUH), King Saud University, Riyadh, Saudi Arabia, from October 2018 to February 2019.

\section{Inclusion criteria:}

The inclusion criteria for SET program in ILD has been broad and participants have had a wide range of ILD diagnoses (Lung fibrosis, Rhumatoid arthtitis, Sclerodema, Dermatomyositis, Ankylosing spondylitis). The patients with stable condition, nonsmokers, having dyspnea on exertion, on medical treatment and not involved in any exercise program in the last 12 months were included. All subjects participated in this study were free from any serious risk of exercise training according to the guidelines of ACC/AHA for exercise testing, American College of Sports Medicine Guidelines for Exercise Testing and Prescription. ${ }^{16}$ Interstitial Lung Disease patients were referred from pulmonary clinic at King Khalid University Hospital (KKUH) and Controls were recruited from the staff at the King Saud University. The control subjects were medically free, nonsmokers and their pulmonary function tests were normal.

\section{Exclusion criteria:}

ILD subjects with long term Oxygen $\left(\mathrm{O}_{2}\right)$ supply, predominant respiratory disease other than ILD, unstable cardiac disease (such as active Ischemic Heart disease, arrhythmia, and pacemaker). Also, subjects who had a musculoskeletal disability (e.g., active arthritis), any comorbidities that prevent exercise, a history of syncope on exertion, communication or transport difficulties and history of arterial $\mathrm{SaO}_{2}$ during exercise (equal or less than $90 \%$ on peak exercise), were all excluded from the study.

Participant having oxygen saturation $\left(\mathrm{SaO}_{2}\right)$ below $90 \%$, required $\mathrm{O}_{2}$ supply. All exercise protocol was supervised and performed with oxygen supplementation $2 \mathrm{~L} /$ Minutes via intranasal cannula to avoid unwanted shortness of breath (SOB). Full written consent obtained from the participants prior 
to the study. Adequate level of confidentiality and privacy of subjects was established. Study protocol was approved by the College of Medicine King Saud University Institutional Review Board (Ref. No. 17/0591/IRB, dated Oct. 5, 2017).

Pulmonary function test(PFT) was carried out by previously recommended techniques. ${ }^{17}$ Measurements of participant's body mass composition, blood pressure, oxygen desaturation SpO2, were recorded before and after the exercise. The endurance exercise training 2 days per week for 8 weeks were carried out by using cycle ergometer and arm-ergometer exercise as described previously. ${ }^{16}$ All outcome measures were determined at baseline (visit 1 ) and after completion of eight weeks of the exercise intervention (visit 15). Following variable indicators were measured before and after SET.

\section{Shortness of breath questionnaire (SOBQ)}

Both patients and healthy subjects were asked to fill SOBQ.) It is a multi-dimensional instrument to measure the three factors of dyspnea (intensity, quality and emotional responses to this sensation). It is a valid and reliable tool used to indicate severity of shortness of breath previously. ${ }^{18}$ The (SOBQ) involves twelve descriptor items on a scale of none (0), mild (1), moderate (2), or severe (3). Total scores from the $(S O B Q)$ range from 0 to 36 , with higher scores showing greater severity.

\section{Six Minutesutes Walk Test (6MWT)}

The 6MWTwas used $^{19}$ for estimating exercise capacity in both ILD patients and controls. The reproducibility of the 6MWD has been confirmed in ILD. ${ }^{19}$ Participants were trained to walk from one end to the other of long uncrowded $50 \mathrm{~m}$ long hallway at their own speed, while trying to cover as much as possible distance in 6 minutes. Finally covering distance was recorded at the end of sixminute's waking before and after SET program.

\section{Heart Rate (HR)}

Heart rate is the speed of the heartbeat measured by the number of contractions (beats) of the heart per minute (bpm). The heart rate can vary according to the body's physical needs, including the need to absorb oxygen and excrete carbon dioxide.Heart rate (HR) of all participants was recorded before and after SET sessions.

Blood Sample Collection: Blood samples in $5 \mathrm{ml}$ EDITA tube (plasma) and $5 \mathrm{ml}$ of plain tube (serum) were collected from patients and healthy controls before and after SET session. Collected blood was stored at $-80{ }^{\circ} \mathrm{C}$ and later used to measure irisin levels by ELISA.
Statistical Analysis: Data were analysed by using the statistical software package SPSS, version 21 and werepresented as mean $\pm S D$. The difference in study variables (6MWT, HR, SOBQ and Irisine levels) in both ILD and control groups were calculated before and after SET. The within and between-group differences were analysed using repeated measures analysis of variance. Between groups comparisons of baseline characteristics were carried out using an unpaired $t$-tesafter checking for normal distribution. The level of significance was set at $p \leq 0.05$. Irisin Protein values were not normally distributed. Mann-Whitney Test was used.

\section{RESULTS}

The impact of eight (8) week SET program on 6MWT, SOBQ HRandIrisine levels were measured before and after each exercise session. Participants were from different age group and sample below and above 40 years shows significant difference $(\mathrm{p}=0.008)$ however body fat percent values, as quantified by body-mass index (BMI) $(\mathrm{kg} / \mathrm{m} 2)$ were found non-significant $(\mathrm{p}=0.228)$.

Six Minutesutes' walk test (6MWT) results of the sampled study groups are shown in Table-I. All patients with ILDand controls improved 6MWD score after SET program. Mean distance of $6 \mathrm{MWT}$ in ILD patientsand controls increased significantly at 15 visit. However, 6MWT scorefound significantly higher in controls than ILD patients.

Trends in HR beforeand afterSETrespectively at each visit among the study groups are shown in Fig-1 and II. Patients group comparatively has higher HR than controls before starting SET (Fig.1) and after completing SET (Fig.2). Table-II shows overall change (difference between pre and post SET) in heart rate (HR) ofILD patents (30-35 bpm) compared tocontrols (40-45 bpm) at each visit.

Table-I: Six Minutesutes' walk test (6MWT) of the sampled study groups.

\begin{tabular}{llccc}
\hline 6MWTdistance & \multicolumn{2}{c}{ Group } & $p^{1}$ \\
\cline { 3 - 4 } (meter) & & $\begin{array}{c}\text { Patient } \\
(n=10)\end{array}$ & $\begin{array}{c}\text { Control } \\
(n=18)\end{array}$ & \\
\hline Visit 1 & Range & $300-550$ & $392-656$ & $0.001^{*}$ \\
& Mean (SD) & $395(68.4)$ & $517.4(84.1)$ & \\
Visit 15 & Range & $350-600$ & $420-670$ & $0.01^{*}$ \\
& Mean (SD) & $458.8(87.1)$ & $561.7(81.6)$ & \\
$\mathrm{p}^{2}$ & & $0.001^{*}$ & 0.001 & \\
\hline
\end{tabular}

$\mathrm{p}^{1}$ : adjusted $\mathrm{p}$ value of independent $\mathrm{t}$-testbetween control and patient group.

$\mathrm{p}^{2}$ : adjusted $\mathrm{p}$ value of paired $\mathrm{t}$-testbetween same group. ${ }^{*} \mathrm{p}<0.05$ (significant). 


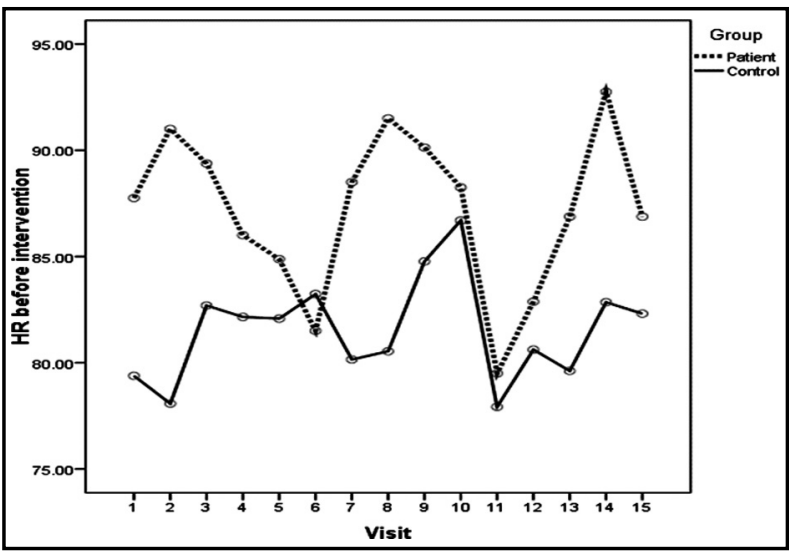

Fig.1: Trend of HR(difference between pre and post SET) before intervention at each visit among the study groups.

Patients with ILD had higher Score of overall SOBQ than controls and the score reduced significantly $(\mathrm{p}=0.012)$ after SET, however overall SOBQ score of control group did not changed remarkably $(\mathrm{p}=0.239)$.

Table-III shows Irisin levelsofILD patientswhich did not changed significantly after SET. Furthermore, there was no significant difference

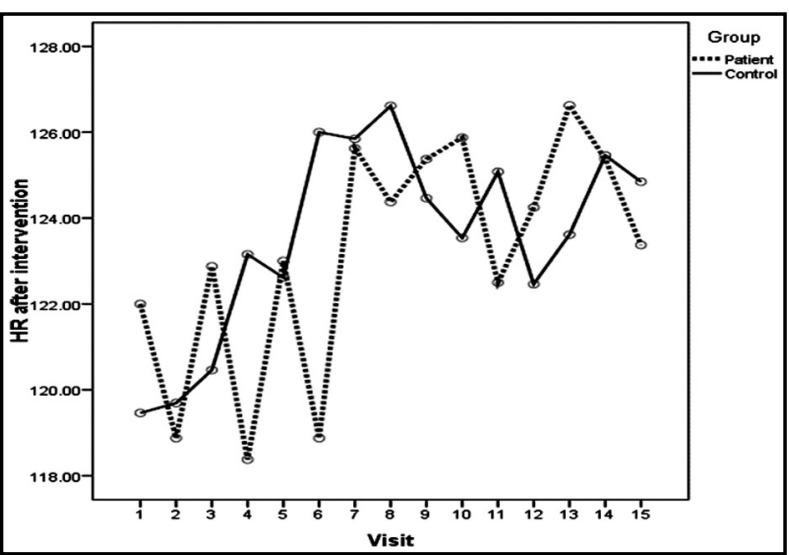

Fig.2: Trend of HR (difference between pre and post SET) after intervention at each visit among the study groups.

in pre-exercise Irisin levels and post exercise Irisin levels of patient and control groups was observed. However, these values mayhave consideredclinically significant.

\section{DISCUSSION}

This is the first interventional design (single-arm clinical trial) studyof SET in ILD patients in Kingdom

Table-II: Overall Change (difference between pre and post SET) in Heart rate (HR) in the study groups.

\begin{tabular}{lccccccc}
\hline & \multicolumn{2}{c}{ HR (bpm) of ILD patients $(n=10)$} & HR ((bpm) of Controls $(n=18)$ & \\
\cline { 2 - 6 } Visit & Pre SET & Post SET & Change in HR & Pre SET & Post SET & Change in HR & \\
& mean (SD) & mean (SD) & mean (SD) & mean (SD) & mean (SD) & mean (SD) & \\
\hline 1 & $85.1(10.1)$ & $117.4(14.7)$ & $32.30(13.22)$ & $81.9(10.1)$ & $124.2(13.1)$ & $42.28(11.79)$ & $0.050^{*}$ \\
2 & $88.6(7.6)$ & $115.5(10.6)$ & $26.90(9.05)$ & $80.1(10.3)$ & $122.4(10.3)$ & $42.33(9.85)$ & $0.001^{*}$ \\
3 & $90.3(13.6)$ & $121.9(8.4)$ & $31.56(18.84)$ & $84.2(9.8)$ & $123.3(12.8)$ & $39.17(14.67)$ & 0.258 \\
4 & $85.8(9.4)$ & $117.4(7.7)$ & $31.67(12.61)$ & $83.3(10.9)$ & $125.4(12.0)$ & $42.06(11.58)$ & $0.045^{*}$ \\
5 & $84.3(9.7)$ & $122.4(5.2)$ & $38.11(8.75)$ & $82.8(11.5)$ & $125.4(13.4)$ & $42.63(13.71)$ & 0.384 \\
6 & $72.4(28.4)$ & $118.9(6.8)$ & $37.38(11.26)$ & $83.4(7.3)$ & $127.0(12.0)$ & $43.64(11.42)$ & 0.228 \\
7 & $88.5(11.6)$ & $125.6(6.3)$ & $37.13(13.16)$ & $80.2(7.0)$ & $125.8(9.9)$ & $45.69(14.02)$ & 0.180 \\
8 & $91.5(5.5)$ & $124.4(8.4)$ & $32.88(11.53)$ & $80.5(10.1)$ & $126.6(9.5)$ & $46.08(12.53)$ & $0.026^{*}$ \\
9 & $90.1(8.5)$ & $125.4(9.5$ & $35.25(8.24)$ & $84.8(8.1)$ & $124.5(6.9)$ & $39.69(5.11)$ & 0.141 \\
10 & $88.3(9.6)$ & $125.9(9.0)$ & $37.63(9.15)$ & $86.7(8.9)$ & $123.5(12.2)$ & $36.85(12.14)$ & 0.878 \\
11 & $79.5(9.6)$ & $122.5(7.2)$ & $43.00(11,74)$ & $77.9(7.5)$ & $125.1(10.3)$ & $47.15(9.52)$ & 0.385 \\
12 & $82.9(6.6)$ & $124.3(9.4)$ & $41.38(12.98)$ & $80.6(5.5)$ & $122.5(8.1)$ & $41.85(11.58)$ & 0.932 \\
13 & $86.9(9.5)$ & $126.6(8.7)$ & $39.75(8.29)$ & $79.6(7.8)$ & $123.6(11.6)$ & $44.00(12.88)$ & 0.417 \\
14 & $92.8(4.9)$ & $125.4(5.8)$ & $32.63(5.78)$ & $82.8(6.8)$ & $125.5(6.9)$ & $42.62(7.42)$ & $0.004^{*}$ \\
15 & $86.9(8.7)$ & $123.38(6.4)$ & $36.50(11.25)$ & $82.3(7.3)$ & $124.85(9.14)$ & $42.54(8.08)$ & 0.168 \\
\hline
\end{tabular}

${ }^{*} \mathrm{p}<0.05$ (significant). 
Table-III: Change in Irisine levels before and after SET among the studygroups.

\begin{tabular}{clccc}
\hline Factor & & \multicolumn{2}{c}{ Group } & $p^{1}$ \\
\cline { 3 - 4 } & & Patient & Control & \\
\hline IRISIN & Range & $1.91-6.78$ & $1.09-4.80$ & 0.22 \\
Before & Mean (SD) & $4.24(1.73)$ & $3.43(1.04)$ & \\
IRISIN & Range & $2.45-8.74$ & $1.35-5.71$ & 0.18 \\
After & Mean (SD) & $4.48(2.02)$ & $3.39(1.41)$ & \\
$\mathrm{p}^{2}$ & & 0.68 & 0.09 & \\
\hline
\end{tabular}

$\mathrm{p}^{1}$ : adjusted $\mathrm{p}$ value of independent t-testbetween control and patient group.

$\mathrm{p}^{2}$ : adjusted $\mathrm{p}$ value of paired t-testbetween same groups.

${ }^{*} \mathrm{p}<0.05$ (significant).

of Saudi Arabia.In this study all patients (male=3, female $=7$ ) with IL improved 6MWT significantly which is consistent with earlier reports, showed a significant improvement in 6MWD following pulmonary rehabilitation in ILD patients. ${ }^{6}$ Our results showed the overall mean improvement of $53 \mathrm{~m}$ in $6 \mathrm{MWD}$ which was greater than the minimal important difference (MID) (30-33 m) and previously reported, $(35 \mathrm{~m})$ for subjects with ILD. ${ }^{5}$ Though increases in 6MWD beyond the $\mathrm{MCID}^{6}$ have been described after exercise training (ET) in patients with ILD, however, the mechanism by which this increase wasarbitrated was not known. Moreover, our results suggested that clinically relevant improvement in symptoms did occur in most participants following exercise training.6MWT distance is a possibly useful biomarker of chronic obstructive pulmonary disease (COPD) severity, which is influenced by muscle weakness, pulmonary vascular disease. ${ }^{20}$ Thedetected improvement in 6-MWD was assumed to be clinically significant. Moreover, causes or severity of ILD did not predict temporary improvement in $6 \mathrm{MWD}$ or signs. This recommends that exercise training may be effective throughout the entire scale of disease in succeeding short-term benefits. Therefore, all patients with ILD should be provided with the chance to carry out exercise training, though timely referral is suggested to support longer-permanent effects. Other approaches such as a longer-term intervention, maintenance exercise programmes or regular sessions of exercise training may also support longer-lasting benefits but further research is required in this main region.

In spite of the limited improvement in those with ILD, some symptomatic benefit was attained with variations in SOBQ and HR symptoms beyond the MCID as 53. Generally, the SOBQ and HR score improved after exercise. Further investigation is necessary to explain the usefulness of the SOBQ in determining change in dyspnoea in ILD. These facts have important clinical significance and practical associations, since proven and effective therapies for ILD are restricted.

"Over 200 etiologies can result in ILD which is identified by irreversible fibrotic reorganization of the lung parenchyma. Interstitial fibrosis creates a barrier to gas exchange at the alveolar-capillary interface, and decreases lung dispensability. "Consequently, hemoglobin oxygenation is reduced and the work of ventilation is increased, leading to reduced cardiorespiratory function. ${ }^{21} \mathrm{In}$ addition to low cardiorespiratory capacity, skeletal muscle weakness has also been linked with poor $6 \mathrm{MWT}$ performance in patients with ILD, which is the most often used method for assessing exercise capacity in these ILD patients. ${ }^{22}$

The impact of SET on development of physiological and clinical results in ILD can be described by numerous mechanisms. ILD patients generally show compromised lung function and inefficient breathing patterns as part of the restrictive pathophysiology. ${ }^{23}$ It is likely that repetitive stimulus of high ventilatory stresses throughout exercise periods, chest expansion during deep breathing exercises and stretching of the thoracic muscles that were used in our program resulted in a more efficient breathing pattern, recovered strength of respiratory muscles, improved pleural elasticity and pulmonary compliance within the lung tissue, and decreased dyspnea perception following the SET program. ${ }^{23}$ SET program duration has been under debit. The perfect duration of pulmonary rehabilitation (PR) for people with ILD is uncertain. The British guidelines for PR suggested programs of 6-12 weeks 'length, but no recommendations particular to ILD were made. ${ }^{25}$

Our results showed no significant difference between the concentration of pre-exercise Irisin level and post exercise level in patient as well as in control groups. However, patients Irisin values were higher than control group which may considered clinically significant.

Several studies have examined the effect of exercise on Irisin concentration and have reported contradictory results. ${ }^{25}$ Various studies using different physical exercise methods have also failed to identify the link between Irisin levels and exercise. ${ }^{25}$ However, various studies have shown an increase in circulating levels of Irisin after exercise training. ${ }^{26}$ 
In humans, the period of exercise appears to be important for variations in circulating levels of Irisin ${ }^{12}$ The type of acute exercise might affect Irisin, some studies suggested that aerobic exercise, as well as other resistance exercises or heavy strength training, stimulates the increase in circulating levels of Irisin. ${ }^{26}$ Severalessential questions remain to be answered. As in vitro muscle contraction does not stimulate the release of Irisin, the high serum levels of the hormone that are observed after acute exercise might be the result of muscle damage or of unidentified biochemical and molecular changes. ${ }^{27}$

It was assumed ${ }^{28}$ that Irisin should respond to several acute and chronic exercise protocols. On the other hand, whether an exercise induced effect on Irisin parameteroccurs or not persists a rather debatable subject, since experimental data differ between studies giving rise to a discussion that emphases on:

1. Whether Irisin is essential in human metabolism.

2. Whether exercise is an effective incentive for Irisin release.

3. What are the physiological effects of irisin in human exercise?

4. Whether exercise encourages FNDC5 expression in different organs.

5. The suitability of the existing techniques to measure circulating irisin in human samples.

In spite of strong indication for the advantages of SET in ILD, this area remains to develop. Essential concerns still remain to be answered including the best design and content for SET; techniques to cover period of advantage; and how SET can best be customized to the multifaceted requirements of subjects with ILD. For example, the best approaches to offer complete SET for subjects with advanced or last-stage disease have not been investigated. Additional work required to describe the non-exercise factors of SET that provide to best results, including psychosocial support, education, behavioral intervention and group therapy.

Limitations of the study:The small sample size and heterogeneous sample group with respect to inclusion of patients with numerous ILD etiologies prevent the capability to generalize our results. Due to the importance of promoting physical health in ILD patients, further investigations in large randomized controlled studies are required with different training modalities to optimize the SET programmes for ILD.

In summary, current studies recommended that SET is effective through the spectrum of ILD and could be offered to all patients who are symptomatic on exertion, irrespective of underlying diagnosis. Although the clinical effectiveness of SET in ILD is increasingly understood, clarification of cost effectiveness of SET would offer motivation for commissioners and policy makers to confirm this intervention is generally available. ${ }^{29}$ The benefit of exercise training could differ according to disease severity and aetiologyand the tiMinutesg of exercise training may matter for particular types of ILD. Due to the importance of promoting physicalhealthinILD patients, further investigation in large randomized controlled studies should address different training modalities to optimize the exercise training programs for ILD.

\section{CONCLUSION}

This study demonstrates that the SET program is safe and effective for patients with ILD, delivering clinically important improvements in dyspnea and exercise capacity. This strengthens the recommendation for the role of SET as a part of a standard comprehensive treatment for ILD patients.Our results showed that SET does not affect circulating Irisin levels in ILD patients. However, further study is needed on large scale to assess the physiological impact of the Irisin in in ILD patients and also confirm or refute a role of Irisin in exercise-induced energy metabolism.

Acknowledgement: The authors would like to express gratitude to the College of medicine Research Center, Deanship of Scientific Research, King Saud University, Riyadh, Saudi Arabia for sponsoring and supporting this study.

Declaration of interest: The authors declare that they have no conflict of interest.

\section{REFERENCES}

1. Saxton JM. Exercise and Chronic Disease: An EvidenceBased Approach. London, Routledge, 2011.

2. Sharp C, McCabe M, Hussain MJ, Dodd JW, Lamb H, Adamali $\mathrm{H}$, et al. Duration of benefit following completion of pulmonary rehabilitation in interstitial lung disease an observational study. QJM. 2017;110:17-22. doi: 10.1093/ qjmed/hcw105

3. Nakazawa A, Cox NS, Holland AE. Current best practice in rehabilitation in interstitial lung disease. Ther Adv Respir Dis. 2017;11(2):115-128.doi: 10.1177/1753465816676048

4. Spruit MA, Pitta F, Garvey C, Zu Wallack RL, Roberts CM, Collins EG, et al. Differences in content and organisational aspects of pulmonary rehabilitation programmes.Eur Respir J. 2014;43(5):1326-1337.doi: 10.1183/09031936.00145613 
5. Holland AE, Hill CJ, Conron M, Munro P, McDonald CF. Short term improvement in exercise capacity and symptoms following exercise training in interstitial lung disease. Thorax. 2008;63:549-554.doi: 10.1136/thx.2007.088070

6. Holland AE, Hill CJ, Glaspole I, Goh N, McDonald CF. Predictors of benefit following pulmonary rehabilitation for interstitial lung disease. Respir Med. 2012;106:429-435. doi: 10.1016/j.rmed.2011.11.014

7. Vainshelboim B, Oliveira J, Yehoshua L, Weiss I, Fox BD, Fruchter $\mathrm{O}$, et al. Exercise training-based pulmonary rehabilitation program is clinically beneficial for idiopathic pulmonary fibrosis. Respiration. 2014; 88:378-88.doi: 10.1159/000367899

8. Bonini M, Fiorenzano G. Exertional dyspnoea in interstitial lung diseases: the clinical utility of cardiopulmonary exercise testing. Eur Respir Rev. 2017;26(143) doi: 10.1183/16000617.0099-2016

9. Dowman L, Hill CJ, Holland AE. Pulmonary rehabilitation for interstitial lung disease. Cochrane Database Syst Rev. 2014 Oct 6 ;(10):CD006322. doi: 10.1002/14651858.

10. Raghu G, Collard HR, Egan JJ, Martinez FJ, Behr J, Brown KK, et al. An official ATS/ERS/JRS/ALAT statement: idiopathic pulmonary fibrosis: evidencebased guidelines for diagnosis and management. Am J Respir Crit Care Med. 2011;183:788-824.doi: 10.1164/rccm.2009-040GL

11. BiniaMinutesov N, Bandt S, Roth A, Haertel S, Neumann $\mathrm{R}$, Bub A. Irisin, physical activity and fitness status in healthy humans: No association under resting conditions in a cross-sectional study. PLoS One. 2018;13(1):e0189254. doi: 10.1371/journal.pone.0189254

12. Huh JY, Mougios V, Skraparlis A, Kabasakalis A, Mantzoros CS. Irisin in response to acute and chronic whole-body vibration exercise in humans. Metabolism. 2014;63(7):918921.doi: $10.1016 /$ j.metabol.2014.04.001

13. Moreno-Navarrete JM, Ortega F, Serrano M, Guerra E, Pardo G, Tinahones F, et al. Irisin is expressed and produced by human muscle and adipose tissue in association with obesity and insulin resistance. JClinEndocrinolMetab. 2013;98(4):E769-E78.doi: 10.1210/jc.2012-2749

14. Nygaard H, Slettalokken G, Vegge G, Hollan I, Whist JE, Strand $\mathrm{T}$, et al. Irisin in blood increases transiently after single sessions of intense endurance exercise and heavy strength training. PLoS One. 2015;10:e0121367. doi: 10.1371/ journal.pone. 0121367

15. Norheim F, Langleite TM, Hjorth M, Holen T, Kielland A, Stadheim HK, et al. The effects of acute and chronic exercise on PGC-1alpha, irisin and browning of subcutaneous adipose tissue in humans. FEBS J. 2014;281(3):739-749. doi: 10.1111/febs.12619

16. Medicine ACoS.ACSM's guidelines for exercise testing and prescription: Lippincott Williams\&Wilkins; 2013.

17. Gardner R, Hankinson J, Clausen JL, Crapo RO, Johnson EL Jr Epler CR XFS. Statement on standardization of spirometry-1987 update. Am Rev Respir Dis. 1987;136:1285-1298.

18. Alyami MM, Jenkins SC, Lababidi H, Hill K. Reliability and validity of an Arabic version of the dyspnea-12 questionnaire for Saudi nationals with chronic obstructive pulmonary disease. AnnThoracMed. 2015;10(2):112-117. doi: $10.4103 / 1817-1737.150730$

19. Singh S, Puhan M, Andrianopoulos V, Hernandes N, Mitchell K, Hill C, et al. An official systematic review of the European Respiratory Society/American Thoracic Society: measurement properties of field walking tests in chronic respiratory disease. Eur Respir J. 2014;44:1447-1478. doi: 10.1183/09031936.00150414
20. Spruit MA, Polkey MI, Celli B, Edwards LD, Watkins ML, Pinto-Plata V, et al. Predicting outcomes from 6-Minutesute walk distance in chronic obstructive pulmonary disease. J Am Med Dir Assoc. 2012;13:291-297. doi: 10.1016/j. jamda.2011.06.009

21. Donner CF, Carone M, Patessio A, Appendini L. Cardiopulmonary exercise testing in interstitial lung disease. Z Kardiol. 1994;83(Suppl 3):159-162.

22. Holland AE, Hill CJ, Conron M, Munro P, McDonald CF. Small changes in six-Minutesute walk distance are important in diffuse parenchymal lung disease. Respir Med. 2009;103:1430-1435.doi: 10.1016/j.rmed.2009.04.024

23. American Thoracic Society (ATS), European Respiratory Society (ERS): Idiopathic pulmonary fibrosis: diagnosis and treatment - international consensus statement. Am J Respir Crit Care Med. 2000;161:646-664.doi: 10.1164/ ajrccm.161.2.ats3-00

24. Bolton C, Bevan-Smith E, Blakey J, Crowe P, Elkin S, Garrod $\mathrm{R}$, et al. Commentary: British thoracic society guideline on pulmonary rehabilitation in adults. Thorax. 2013;68(9):887888. doi: 10.1136/thoraxjnl-2013-203809

25. Kurdiova T, Balaz M, Mayer A, Maderova D, Belan V, Wolfrum $\mathrm{C}$, et al.Exercise-mimicking treatment fails to increase Fndc5 mRNA and irisin secretion in primary human myotubes. Peptides. 2014;56:1-7. doi: 10.1016/j. peptides.2014.03.003

26. Loffler D, Muller U, Scheuermann K, Friebe D, Gesing J, Bielitz J, et al. Serum irisin levels are regulated by acute strenuous exercise. J Clin Endocrinol Metab. 2015;100(4):1289-1299.doi: 10.1210/jc.2014-2932

27. Perakakis N, Triantafyllou GA, Fernandez-Real JM, Huh JY, Park KH, Seufert J, et al. Physiology and role of irisin in glucose homeostasis. Nat Rev Endocrinol. 2017;13(6):324337.doi: $10.1038 /$ nrendo.2016.221

28. Fatouros IG. Is irisin the new player in exercise-induced adaptations or not? A 2017 update. Clin Chem Lab Med. 2018;56(4):525-548.doi: 10.1515/cclm-2017-0674

29. Loveman, E, Copley V, Colquitt J, Scott, D, Clegg A, Jones J, et al. The effectiveness and cost-effectiveness of treatments for idiopathic pulmonary fibrosis: systematic review, network meta-analysis and health economic evaluation. BMC Pharmacol Toxicol. 2014;15:63. doi: 10.1186/20506511-15-63.

\section{Authors' Contribution:}

RMA: Did the data analysis and data interpretation. AMA:Responsible and accountable for the accuracy or integrity of the work.

Conceived the idea and designed the study. Approval of the final version to be published.

ARA:Drafting and revising the paper.

GAN: Literature search and final review.

Authors:

1. Rahma Mohammad Alyami, MS

College of Medicine, King Khalid University,

Abha, Saudi Arabia.

2. Abdulrahman Mohammed Alhowikan, PhD.

Department of Physiology, Faculty of Medicine,

3. Abdullah Rashed Alharbi, MBBS

Department of Medicine,

4. Ghada AL-Nafisah, MS.

Research Center,College of Medicine,

2-4: King Saud University, Riyadh, Saudi Arabia. 